\title{
Splitting and merging an elongated Bose-Einstein condensate at finite temperature
}

\author{
A. Mebrahtu, ${ }^{1,2}$, A. Sanpera, ${ }^{1,3}$, and M. Lewenstein ${ }^{1,4}$ \\ 1 Institut für Theoretische Physik, Universität Hannover, D-30167 Hannover, Germany \\ ${ }^{2}$ Department of Physics, Mekelle University, P. O. Box 231, Mekelle, Ethiopia. \\ ${ }^{3}$ ICREA and Grup de Física Teòrica, Universitat Autònoma de Barcelona, E-08193 Bellaterra, Spain. \\ 4 ICREA and ICFO-Institut de Ciències Fotòniques, \\ Parc Mediterrani de la Tecnología, E-08860 Barcelona, Spain.
}

\begin{abstract}
We analyze coherence effects during the splitting of a quasi one-dimensional condensate into two spatially separated ones and their subsequent merging into a single condensate. Our analysis takes into account finite-temperature effects, where phase fluctuations play an important role. We show that, at zero-temperature, the two split condensates can be merged into a single one with a negligible phase difference. By increasing temperature to a finite value below the critical point for condensation $\left(T_{c}\right)$, i.e., $0 \leq T / T_{c}<1$, a considerable enhancement of phase and density fluctuations appears during the process of splitting and merging. Our results show that if the process of splitting and merging is sufficiently adiabatic, the whole process is quite insensitive to phase fluctuations and even at high temperatures, a single condensate can be produced.
\end{abstract}

PACS numbers: 03.75.Lm,03.75.Nt,05.30.Jp

\section{INTRODUCTION}

The experimental exploration of Bose-Einstein condensates (BECs) in many different systems such as alkali metal gases 1, 2, 3], hydrogen [4], meta-stable helium [5], and molecules 6, 7, 8] has led to a surge of interest in manipulating ultracold atomic samples under very different circumstances. One of the initial motivations for such development was and is the prospect of creating a continuous and coherent atomic beam 9, 10, 11, which is the atomic analogy of the optical laser beam 12 .

Among the major challenges in achieving a continuous atom laser is how to overcome the difficulty due to the restrictive cooling conditions for continuously condensing the atomic gas. Spatial separation of the evaporative cooling from the destructive laser cooling may play a role in alleviating these challenges [13]. Recently, a continuous BEC source was achieved by periodically replenishing a reservoir with condensates 11]. There, optical tweezers were used to transport sodium condensates from where they were created to the reservoir. Such procedure allows one to continuously replenish the reservoir which contains on average more than $10^{6}$ atoms. Bringing a new condensate to the reservoir each time implies the merging of condensates with different relative phases since each condensates is created with a different phase. The merging of two condensates with different phases poses a limitation on the coherence of the process.

Recently, interference effects in the merging of 30 uncorrelated Bose-Einstein condensates released from a one-dimensional optical lattice have been discussed in [14], whereas coherent splitting of BECs by deforming an optical single-well into a double-well potential for a trapped atom interferometer have been addressed in Refs. [15, 16]. Very recently, Schumm et al. 17] has demonstrated a coherent beam splitter on an atom chip by splitting the condensate in a double-well potential and merging it again. They have demonstrated phase preservation in this process, even in the case when the split BECs are far enough apart to inhibit tunnel coupling.

In this paper, we analyze the axial splitting of a very much elongated cigar-shape condensate into two condensates [18, 19] and their subsequent merging along the axial direction. Our analysis includes finite-temperature effects. In other words, phase fluctuations arising due to temperature are taken into account during the whole process: splitting and merging. We observe that as long as the process of splitting and merging is adiabatic enough, both the split and the merged condensates, even at relatively large temperatures, do survive the effects of these fluctuations.

Low-dimensional quantum gases exhibit very fascinating properties and have attracted a lot of interest, both theoretically and experimentally 20, 21, 22, 23, 24, 25, 26]. It is known that low-dimensional [one- (1D) and two- (2D) dimensional] quantum gases differ qualitatively from their 3D counterparts 21, 27, 28, 29]. From a theoretical point of view, the use of a mean-field theory to describe a low-dimensional quantum gas is severely restricted. A widely used criterion to apply a mean-field approach is to demand that the average distance between particles, $\mathrm{d}$, is clearly smaller than the correlation length of the condensate $l_{c}=\hbar / \sqrt{m n g}$ where $m, g$, and $n$ denote the mass, the interaction coupling, and the density, respectively. In three dimensions, the above condition leads to $l_{c} / d \propto n^{-1 / 6}$ and is well satisfied for small densities, and the description of the system at $T=0$ with a mean-field Gross-Pitaevskii equation is fully satisfactory. In the one-dimensional case, however, this ratio behaves as $l_{c} / d \propto n^{1 / 2}$ and this fact changes drastically the range of applicability of a mean-field treatment. A careful analysis of the validity of a mean-field treatment in the $1 \mathrm{D}$ 
case [30] leads to the following condition:

$$
\left(\frac{N a_{s} a_{z}}{a_{\perp}^{2}}\right)^{1 / 3} \gg 1
$$

where $N$ is the number of condensed atoms, $a_{z}=$ $\sqrt{\hbar /\left(m \omega_{z}\right)}$ and $a_{\perp}=\sqrt{\hbar /\left(m \omega_{\perp}\right)}$ are the axial and radial oscillator lengths, respectively, and $\omega_{z}$ and $\omega_{\perp}$ are the angular frequencies in the corresponding directions. Thus, in 1D, contrary to the 3D case, when the density decreases the gas gradually becomes strongly correlated, acquires a fermionic character, and enters into the socalled Tonks-Girardeau regime [31, 32, 33, 34]. Experimental demonstration of a Tonks gas has been recently achieved 24].

The possibility of generating low-dimensional bosonic gases raises the question of the effects of quantum fluctuations. In an untrapped 1D Bose system these fluctuations destroy finite- as well as zero-temperature condensation. For trapped Bose gases, the situation is quite different: for noninteracting bosons in such a trap the finite spacing between the lowest and the next energy level allows for the occurrence of 1D Bose-Einstein condensation even at finite-temperatures as stipulated in Refs. 20, 21]. In such a case the absence of gapless excitations indicates that the BEC will not be destroyed immediately as interactions between bosons are turned on.

In 1D trapping geometries, long-wavelength density and phase fluctuations lead to a new intermediate state between a condensate and a noncondensed system, which is commonly referred to as a quasicondensate. In quasicondensates, the phase of the condensate is only coherent over a finite distance that is smaller than the system size. In other words, the phase coherence length is smaller than the axial size of the sample. To understand the nature of quasicondensates at finite-temperature, one has to analyze the behavior of the single particle correlation function by calculating the fluctuations of phase and density as has been done by Petrov et al. 21]. There it is shown that for temperatures below the degeneracy temperature, the condensate's phase indeed fluctuates, but fluctuations of the density are still highly suppressed. This character of thermal fluctuations is also present in highly elongated 3D gases 35], and has been recently observed experimentally in $36,37,38,39,40,41$.

Quasi 1D geometries can be accessible in magnetic traps with a cylindrically symmetric harmonic potential along the axial direction that have transverse frequencies $\omega_{\perp}$ much larger than the axial one $\omega_{z}$. In such configurations the resulting condensate looks like a cigar aligned along the symmetry or $\mathrm{z}$ axis. With current technology, condensates with aspect ratio $\lambda=\omega_{\perp} / \omega_{z}$ as large as 1000 are achievable.

In this paper, we study the process of splitting and subsequent merging of an elongated condensate confined in a $1 \mathrm{D}$ geometry both at $T=0$, i.e., when the condensate has a well-defined phase, and at finite-temperature
$(T>0)$, in the quasicondensate regime. For finite $T$, we analyze the process of splitting and merging for a wide range of temperatures, i.e., $T_{\phi}<T<T_{d}$, where $T_{\phi}=T_{d} \hbar \omega_{z} / \mu$ ( $\mu$ being the chemical potential) corresponds to the characteristic temperature above which a true-condensate turns into a quasicondensate in which phase fluctuations begin to play a role. On the other hand $T_{d}=N \hbar \omega_{z} / k_{B}$ is the $1 \mathrm{D}$ degeneracy temperature 21]. The transition, or crossover, between the different regimes for the 1D degenerate interacting bosonic gas, i.e., true-condensate, quasi-condensate, and Tonks gas is smooth. Thus, in the regime of quasi-condensation density fluctuations are relatively suppressed while phase fluctuations are enhanced. By keeping all parameters fixed and reducing the number of atoms, phase fluctuations become more and more pronounced, mean-field theory fails, and the gas enters into the strongly correlated regime or Tonks gas 31, 32, 33, 34].

In our case, the splitting of the condensate is achieved by means of a double-well potential grown adiabatically on top of a trapping harmonic potential. By adiabatically switching off the double-well potential, we merge these condensates into a single one (Merged BEC). We would like to stress that we use the mean-field GrossPitaevskii equation (GPE) throughout this paper. It is worth pointing out that although the Gross-Pitaevskii equation [42] describes properly coherent evolution of the atomic mean-field at $T=0$, it can also be used to solve time evolution at finite-temperature in a relatively straightforward manner. It might look like that the mean-field method of the GPE allows to make statements only about first order coherence. But, as it is well known, a closely inspection reveals that the GPE contains as such classical Bogoliubov-de Gennes (BdG) equations, i.e. equations describing small fluctuations around a given solution of the GPE. Since we are here interested in the study of relatively high temperatures $T>T_{\phi}$, classical description of fluctuations is fully appropriate. It turns out that one can simulate temperature effects by adding fluctuations to the ground state solution of the GPE at $T=0$ in a way which mimics thermal fluctuations. At low temperatures $T<T_{c}$, where $T_{c}=N / \ln (2 N) \hbar \omega_{z} / k_{B}$ [20], this can be generally done by identifying phonon (quasi-particle) modes, i.e. eigensolutions of the BdG equations. The fluctuations are expressed thus as sums over the quasi-particles with amplitudes taken from Monte Carlo sampling and corresponding to the thermal (Boltzmann) populations of the quasi-particle modes. Such a method is used in this paper, with the additional simplification that for quasi-1D situations only the phase fluctuations are relevant.

Note, that once we add the fluctuations initially at $t=0$, and as long as they remain small in the course of evolution, they will propagate in time with a very good approximation as appropriate solutions of the time dependent BdG equations. Note also, that our approach 
allows in principle to obtain information about all correlation functions: either in the form of an average over Monte Carlo realizations of the initial fluctuations, or in the form of time averages, due to the (expected) ergodic character of the evolution. In some cases, even averaging over the initial data is not necessary: the results for different realizations are so similar that looking for few single cases allows one to draw conclusions about "coherence". By coherence we mean here, that the splitting process of an initial 1D BEC into two spatially separated 1D BECs occurs with a well-defined relative phase between them. Their subsequent merging into a single merged BEC, when the process is fully coherent, should result in a single condensate with a well-defined relative phase with respect to the initial one. If this is the case, there is a perfect overlap between the densities of the initial and merged condensates.

It is worth stressing that our approach is a simplified version of the classical field methods used by several groups [41, 43, 44, 45, 46, 47, 48, 49, 50]. In their approach some emphasis was put on the explanation of the temperature concept, and a certain model of finitetemperature effects has been studied. Particularly interesting is here the possibility of extracting higher order correlations from a single shot measurements 51, 52, 53., which in our case corresponds to a single realizations of the initial fluctuations.

\section{DESCRIPTION OF THE MODEL}

Here we consider ${ }^{87} \mathrm{Rb}$ condensate with $N=1.2 \times 10^{4}$ atoms confined in an harmonic trap with frequencies $\omega_{\perp}=2 \pi \times 715 \mathrm{~Hz}$ and $\omega_{z}=2 \pi \times 5 \mathrm{~Hz}$. For such parameters, the system is in the $1 \mathrm{D}$ Thomas-Fermi regime $\left(\mu \gg \hbar \omega_{z}\right)$ along the axial direction. The 1D chemical potential is given by

$$
\mu=\hbar \omega_{z}\left(\frac{3}{4 \sqrt{2}} \frac{N m g_{1 \mathrm{D}} \sqrt{\hbar / m \omega_{z}}}{\hbar^{2}}\right)^{2 / 3}
$$

where $g_{1 \mathrm{D}}=2 \hbar \omega_{\perp} a_{s}$ is the effective $1 \mathrm{D}$ coupling strength [55]. For our parameters, transverse excitations are suppressed $\left(\mu \simeq \hbar \omega_{\perp}\right)$, and the dynamics of such a Bose gas can be described by the usual mean-field GPE in $1 \mathrm{D}$

$$
\begin{aligned}
i \hbar \frac{\partial \Psi(z, t)}{\partial t} & =\left(-\frac{\hbar^{2}}{2 m} \frac{\partial^{2}}{\partial z^{2}}+V_{T}(z, t)\right. \\
& \left.+g_{1 \mathrm{D}} N|\Psi(z, t)|^{2}\right) \Psi(z, t)
\end{aligned}
$$

where $\Psi(z, t)$ is the mean-field order parameter, or in other words the condensate wave function. The potential term $V_{T}(z, t)$ includes both the magnetic trap and the double-well potential as described below:

$$
\begin{aligned}
V_{T}(z, t) & =V_{\text {trap }}(z)+V_{\mathrm{op}}(z, t) \\
& =\frac{1}{2} m \omega_{z}^{2} z^{2}+S(t) V_{0} \cos \left(k_{l} z\right)^{2} .
\end{aligned}
$$

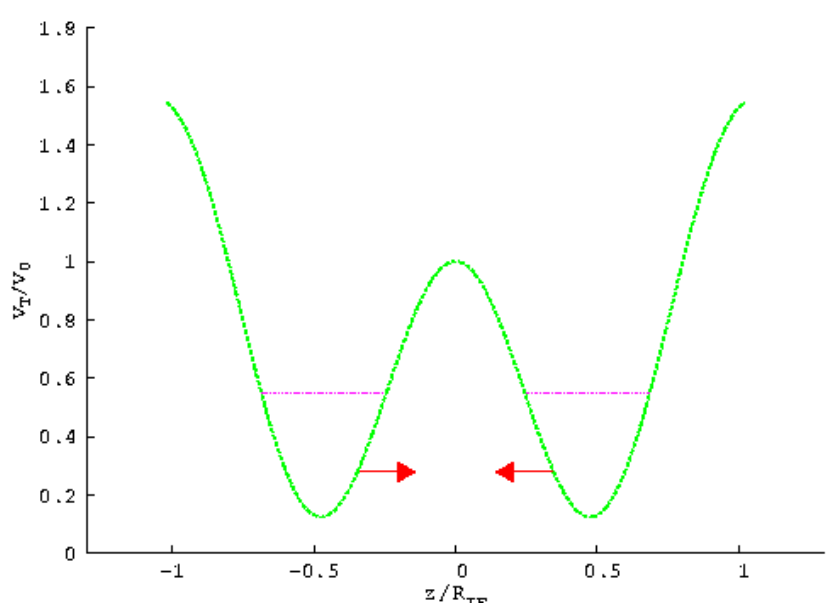

FIG. 1: (Color online) Illustration of a double-well potential for splitting and merging of an elongated 1D BEC: a strong optical potential and an axial trapping potential are combined for creating a double-well potential which is used for splitting a 1D condensate, when switched on adiabatically, into two spatially separated symmetrical $1 \mathrm{D}$ condensates at the center of each well, and then merge them into one when switched off adiabatically again.

The potential used to split the condensate into two spatially separated condensates, $V_{\mathrm{op}}(z, t)$, is switched on and off adiabatically by means of a time-dependent function $S(t)$. The maximum depth of this potential is $V_{0}=2.2 \times$ $10^{4} E_{r}$ in terms of the recoil energy $E_{r}=\hbar^{2} k_{l}^{2} / 2 m$ and $k_{l}=2 \pi / \lambda_{l}$. To achieve spatial separation of the split condensates one has to require that the distance between the two wells is, at least, of the order of the Thomas-Fermi radius $\left(R_{\mathrm{TF}}\right.$.) This radius is given by $R_{\mathrm{TF}}=\sqrt{2 \mu /\left(m \omega_{z}^{2}\right)}$, so that $k_{l}=\pi / R_{\mathrm{TF}}$. For $N \simeq 10^{4}$ atoms, the ThomasFermi radius is $R_{\mathrm{TF}} \simeq 88 \mu \mathrm{m}$. This is in agreement with the experimental results in Refs. 15, 17].

The time dependent function $S(t)$ controls the switching on and off of the double-well potential and hence the overall splitting and merging process of the condensates. We define this function as

$S(t)= \begin{cases}0, & \text { for } t \leq t_{\mathrm{evo}} \text { and } t \geq t_{\mathrm{mer}}, \\ \sin \left(\frac{\pi}{2} \frac{t-t_{\mathrm{ev}}}{t_{\mathrm{spl}}-t_{\mathrm{evo}}}\right)^{2}, & \text { for } t_{\mathrm{evo}}<t<t_{\mathrm{spl}} \\ 1, & \text { for } t_{\mathrm{spl}} \leq t \leq t_{\mathrm{con}} \\ \cos \left(\frac{\pi}{2} \frac{t-t_{\mathrm{con}}}{t_{\mathrm{mer}}-t_{\mathrm{con}}}\right)^{2}, & \text { for } t_{\mathrm{con}}<t<t_{\mathrm{mer}}\end{cases}$

In this equation (5), $t_{\text {evo }}$ is the time duration required for evolving the 1D BEC in real time before the splitting process begins. It extends for $10 \mathrm{~ms}$. Just immediately 
after, the splitting process begins and continues for a time interval $t_{\mathrm{spl}}$. At the end of $t_{\mathrm{spl}}$, the function $S(t)$ attains a maximum value of unity and remains constant for a time interval $t_{\text {con }}$. During this time interval, two spatially separated 1D BECs are created. At the beginning of the time span $t_{\text {mer }}$, merging of the condensates starts by switching off the double-well potential. This process continues until the two 1D BECs merge together. A complete merging is only possible when $S(t)$ becomes finally zero, i.e., when the optical potential is completely switched off. In this case the atoms remain only under the influence of the trapping potential.

To ensure coherence during the process of the switching on and off of the optical potential, the raising of the double-well has to be slow enough to avoid excitations and to allow for quantum tunneling between the two wells. Notice that the relevant time scale of excitations is given by the inverse of the frequency of the trap, in our case $t_{\mathrm{sys}}=2 \pi / \omega_{z}=200 \mathrm{~ms}$. If $t_{\mathrm{spl}}, t_{\mathrm{mer}} \gg t_{\mathrm{sys}}$ we expect coherent splitting and merging. On the other hand, for $t_{\mathrm{spl}}, t_{\mathrm{mer}}$ of the order of $t_{\mathrm{sys}}$ or less, the process creates more and more excitations that cause incoherence. The faster the double-well potential switches on and off the stronger the excitations are. To check our claims we have carried out further numerical simulations for several values of $t_{\mathrm{spl}}$ and $t_{\mathrm{mer}}$ around $t_{\mathrm{sys}}$. In general, we have observed that a coherent process, at $T=0$, is achieved for switching times of $400 \mathrm{~ms}$ (or larger).

The full time dependency of $S(t)$ necessary for the coherent splitting and merging of the condensates based on Eq. (5) is illustrated in Fig. 2]

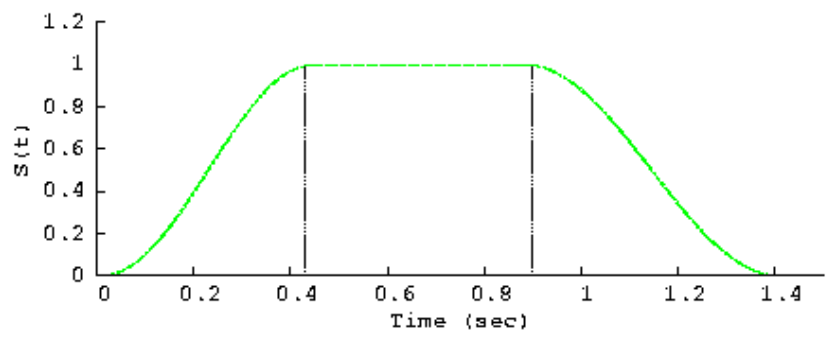

FIG. 2: (Color online) Time dependence of the function $S(t)$. For the whole process, we fixed a time evolution of $t_{\text {evo }}=10$ $\mathrm{ms}$, a splitting time of $t_{\mathrm{spl}}=440 \mathrm{~ms}$, a time $t_{\mathrm{con}}=430 \mathrm{~ms}$ in which $S(t)$ remains constant (unity), and a time of merging $t_{\mathrm{mer}}=530 \mathrm{~ms}$ and an additional time of $10 \mathrm{~ms}$ for allowing the merged condensate to make its final evolution into a single condensate.

\section{SPLITTING AND MERGING AT ZERO TEMPERATURE}

We calculate first the ground state of the system by evolving the GPE (3) with $S(t)=0$ in imaginary time.
After the ground state has been found, we numerically solve Eq. (3) with the pulse profile given by the time dependent-function $S(t)$.

The results of our simulations at temperature $T=0$, are summarized in Fig. 3 There we display the condensates' density at three different times. First at $t=0$, the initial condensate (Initial 1D BEC) has a characteristic Thomas-Fermi profile with $R_{\mathrm{TF}}=88 \mu \mathrm{m}$. Then at $t=800 \mathrm{~ms}$ (corresponding to the ramping up of the double-well potential in $400 \mathrm{~ms}$ and keeping it constant during additional $400 \mathrm{~ms}$ ), two spatially well separated identical condensates (Split BECs) appeared, centered at $z / R_{\mathrm{TF}}= \pm 0.5$, each of them with a number of atoms $N_{s}=N / 2$. Finally, we display the density of the condensate at $t=1400 \mathrm{~ms}$. The merged condensate (Merged 1D BEC) has exactly the same profile as the initial one (Initial 1D BEC) and thus they cannot be distinguished in the figure, asserting that the process of splitting and merging is fully coherent.

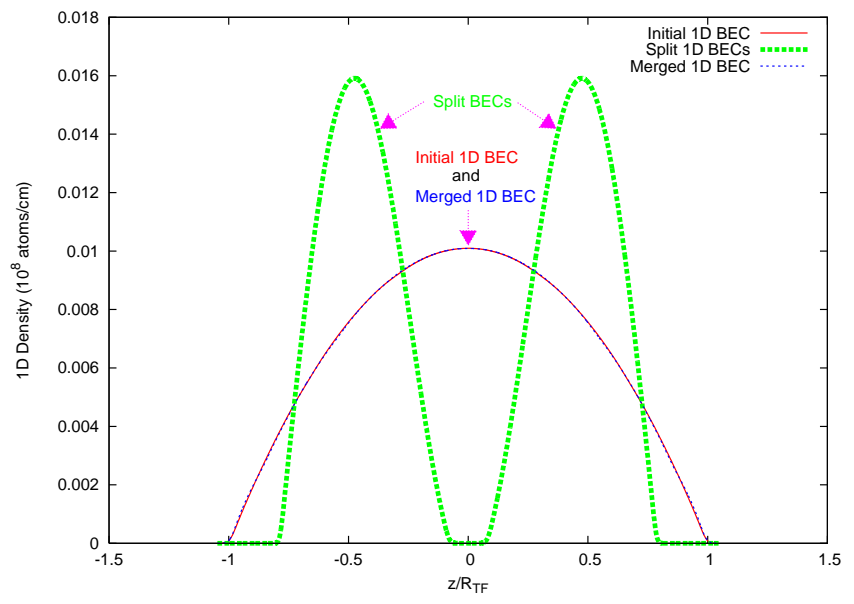

FIG. 3: (Color online) Adiabatic splitting and merging of an elongated condensate at $T=0$. After coherently splitting an initial 1D condensate (Initial BEC) into two spatially separated ones (Split BECs), by adiabatically switching on the double-well potential given in Fig. 1 centered at $z / R_{\mathrm{TF}}=-0.5$ and $z / R_{\mathrm{TF}}=0.5$, the optical potential is again switched off adiabatically. This leads to a coherent merging of the Split condensates into a single one (Merged 1D BEC). The merged 1D BEC overlaps on top of the initial 1D BEC which is an indication of a completely coherent merging process.

Coherence may be a prerequisite for further applications such as in atom interferometry and quantuminformation processing [54].

Before we proceed to the case of finite-temperature, it may be relevant to address the case of a nonadiabatic splitting and merging process, i.e., when the double-well potential switches on and off too fast. We have carried out simulations for switching times as short as $20 \mathrm{~ms}$. In such cases the splitting of the condensate (even at $T=0$ ) becomes completely incoherent, there is no trace of phase 
preservation, and the condensate is destroyed, as is shown in Fig. 4 For shorter splitting times, $t_{\mathrm{spl}}<20 \mathrm{~ms}$, it is

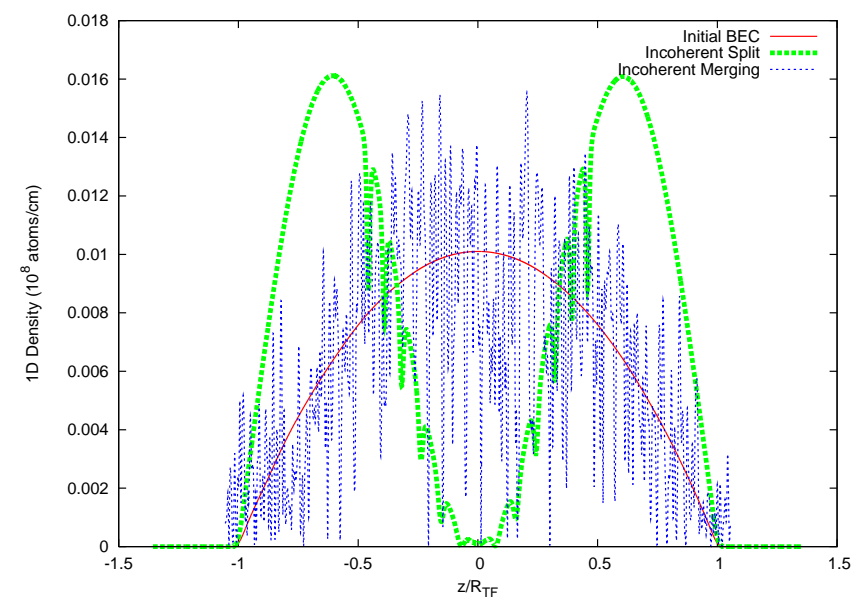

FIG. 4: (Color online) Nonadiabatic splitting and merging of an elongated condensate at $T=0$. The Initial BEC is split incoherently by considering a very small splitting time $\left(t_{\mathrm{spl}}\right)$ into two picked spatially separated ones (Incoherent Split) by nonadiabatically switching on the double-well potential (Fig. 1). The optical potential is again switched off nonadiabatically which leads to a completely incoherent pattern (Incoherent Merging) due to larger excitations.

not even possible to split the Initial BEC into two well spatially separated condensates. When it comes to the time of merging $\left(t_{\text {mer }}\right)$, a similar behavior is observed if $t_{\text {mer }} \leq t_{\text {sys }}$.

\section{SPLITTING AND MERGING AT FINITE TEMPERATURE}

So far we have considered only the case of $T=0$ where the initial condensate has a well-defined phase. In this section, following exactly the same approach as in the previous section, we investigate the effects that finitetemperature might have on the overall coherence during the splitting and merging process.

Fluctuations of phase and density of a BEC are the result of thermal excitations, and appear usually at finitetemperature 35, 38. In such a case the system includes some noncondensed or thermal particles and hence the total Bose field operator can be expressed as

$$
\hat{\Psi}(z, t)=\Psi(z, t)+\delta \hat{\Psi}(z, t),
$$

where $\delta \hat{\Psi}(z, t)$ describes the thermal depletion part. For a BEC in 3D trapping geometries, fluctuations of density and phase are only important in a narrow temperature range near the BEC transition temperature $T_{c}$ 35. For 1D systems, however, phase fluctuations are present at temperatures far below the degeneracy temperature. Phase fluctuations can be studied by solving the Bogoliubov-de Gennes equations describing elementary excitations. Writing the quantum field operator as $\hat{\Psi}(z)=\sqrt{n_{1 \mathrm{D}}(z)} \exp [i \hat{\phi}(z)]$ where $n_{1 \mathrm{D}}(z)=|\Psi(z, t)|^{2}$ denotes the condensate density at $T=0\left[n_{1 \mathrm{D}}(z=0)=\right.$ $\left.\mu / g_{1 \mathrm{D}}\right]$ the phase and density operators take, respectively, the following forms [56]:

$$
\hat{\phi}(z)=\frac{1}{\sqrt{4 n_{1 \mathrm{D}}(z)}} \sum_{j=1}^{\infty}\left[f_{j}^{+}(z) \hat{a}_{j}+f_{j}^{-}(z) \hat{a}_{j}^{\dagger}\right]
$$

and

$$
\hat{n}_{1 \mathrm{D}}(z)=\sqrt{n_{1 \mathrm{D}}(z)} \sum_{j=1}^{\infty} i\left(f_{j}^{-} \hat{a}_{j}-f_{j}^{+} \hat{a}_{j}^{\dagger}\right),
$$

where $\hat{a}_{j}\left(\hat{a}_{j}^{\dagger}\right)$ is the annihilation (creation) operator of the excitations with quantum number $j$ and energy $\epsilon_{j}=$ $\hbar \omega_{z} \sqrt{j(j+1) / 2}$, and $f_{j}^{ \pm}=u_{j} \pm v_{j}$, where $u_{j}$ and $v_{j}$ denote the excitation functions determined by the $\mathrm{BdG}$ equations. More explicitly, the functions $f_{j}^{ \pm}$in a $1 \mathrm{D}$ Thomas-Fermi regime and at finite-temperature take the form:

$$
\begin{aligned}
f_{j}^{ \pm}(z)= & \sqrt{\frac{(j+1 / 2)}{R_{\mathrm{TF}}}}\left(\frac{2 \mu}{\epsilon_{j}}\left[1-\left(z / R_{\mathrm{TF}}\right)^{2}\right]\right)^{ \pm 1 / 2} \\
& \times P_{j}\left(z / R_{\mathrm{TF}}\right)
\end{aligned}
$$

where $P_{j}\left(z / R_{\mathrm{TF}}\right)$ are Legendre polynomials. The phase coherence length, in terms of the Thomas-Fermi radius $R_{\mathrm{TF}}$, is expressed as $L_{\phi}=R_{\mathrm{TF}} T_{d} \mu / T \hbar \omega_{z}$ and characterizes the maximal distance between two phase-correlated points in the condensate. Phase fluctuations increase for large trap aspect ratios and small $N$ [36].

Temperature is included at the level of the GPE (3) by calculating first the density at $T=0$ in the presence of the magnetic trap only, and then mimicking finitetemperature effects via the phase operator of Eq. (7). In other words a phase is imprinted on the condensate wave function at this stage. To this aim, we calculate the Bose occupation $N_{j}=\left(e^{\epsilon_{j} /\left(k_{B} T\right)}-1\right)^{-1}$ modes in the Bogoliubov approximation for fixed temperatures replacing the operators $\hat{a}_{j}$ and $\hat{a}_{j}^{\dagger}$ by random complex variables $\alpha_{j}$ and $\alpha_{j}^{*}$, respectively such that $\left\langle\left|\alpha_{j}\right|^{2}\right\rangle=N_{j}[36]$.

Although the GPE (3) in this limit remains valid, the $\mathrm{BdG}$ equations become modified by the integration over the transverse profile of the condensate [32]. In effect the mode functions $f_{j}^{ \pm}$are given by Jacobi polynomials, whereas $\epsilon_{j}$ are given by a slightly different expression than in the pure $1 \mathrm{D}$ case. We stress, however, that for the regimes of temperatures we consider, there will be no qualitative and practically no quantitative difference between the pure- and quasi-1D results.

The dependence of the magnitude of the phase fluctuations of the condensate on the temperature is shown in Fig. 5] Even though we present here results calculated for a fixed number of condensate atoms $(N)$, it is 
numerically verifiable that the magnitude of the phase fluctuations is inversely proportional to the square root of the number of atoms and hence to the peak density of the condensate as described by Eq. (7). As can be
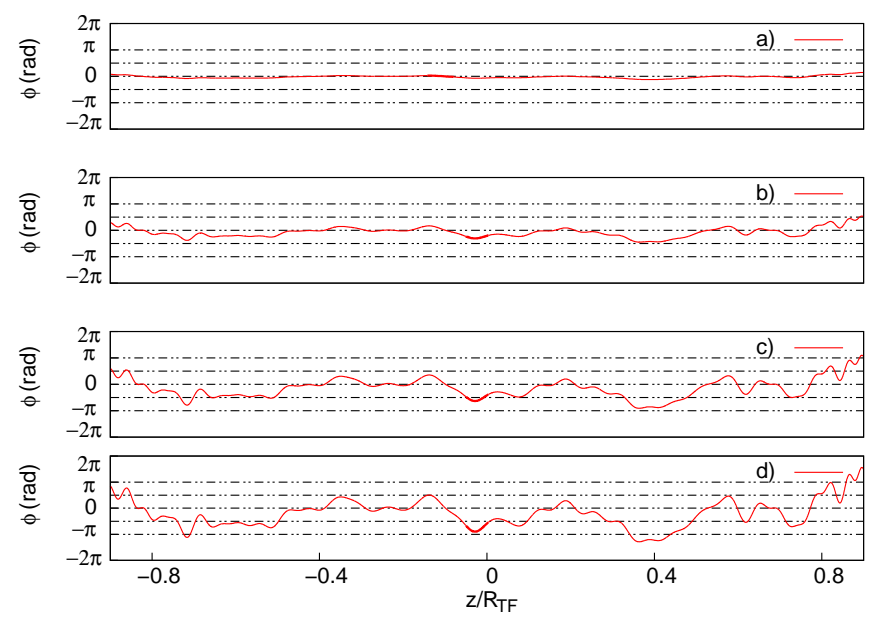

FIG. 5: (Color online) Enhancement of phase fluctuations with the increase in temperature. (a) At the top, we have very weak phase fluctuations at very low temperature $\left(T / T_{c}=\right.$ $0.01)$. (b) With the rise in temperature $\left(T / T_{c}=0.1\right)$, the phase fluctuations begin to be enhanced. (c) Still at an intermediate but relatively high temperature $\left(T / T_{c}=0.4\right)$, the magnitude of phase fluctuations increases. (d) Finally at $T / T_{c}=0.8$, which is near the critical point, stronger phase fluctuations are displayed.

seen by inspecting the different plots (a)-(d) in Fig. [5 the phase fluctuations get more and more enhanced with the increase of temperature.

Having seen the enhancement of phase fluctuations with temperature, we proceed now to analyze the coherence properties of splitting and merging of a $1 \mathrm{D}$ BEC in the presence of these phase fluctuations for temperatures in the range of $T_{\phi}<T<T_{c}$. Our results for finite-temperature are summarized in Fig. [6

Using exactly the same approach that we employed for the adiabatic case at $T=0$ but now including temperature we study again the splitting and merging process for the same parameters. In this case, the density fluctuations, which are highly suppressed in elongated 3D condensates, are very pronounced in the 1D density after time evolution, when the temperature increases from a very small value $\left(T / T_{c}=0.01\right)$ to a value near the critical point for condensation $\left(T / T_{c}=0.8\right)$. At high temperatures, the density fluctuations get more and more enhanced. However, notice that the effect of fluctuations is very similar on the single and on the merged condensates. On the other hand, fluctuations on the split condensates remain relatively small due to fact that the densities in the double wells are higher as shown in all plots of Fig. 6] This is in confirmation of the prediction of Eq. (7). The presence of fluctuations on the density profile is a conse-
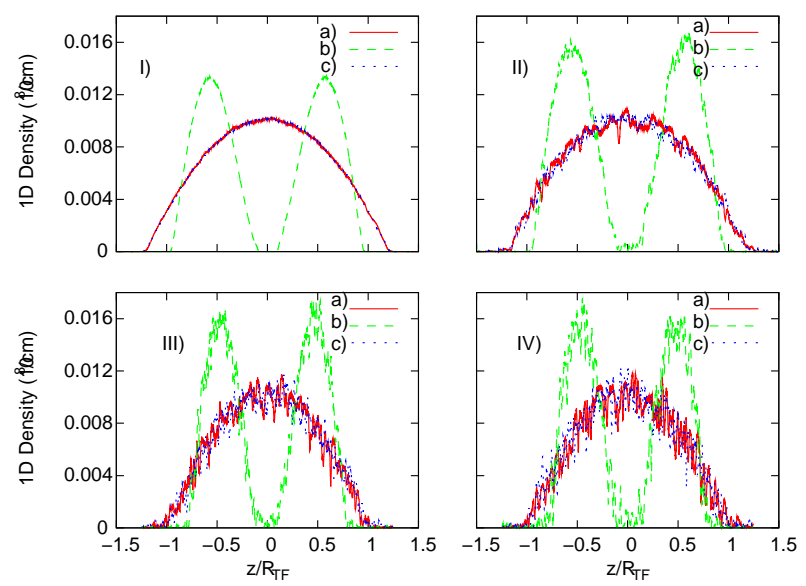

FIG. 6: (Color online) Splitting and merging of 1D BECs at finite-temperature, i.e., in the presence of phase and density fluctuations. The four plots are the fluctuating densities at $T / T_{c}=$ (I) 0.01 , (II) 0.1 , (III) 0.4 and (IV) 0.8. Each one in turn consists three plots with in it: curve a, the initial 1D BEC (red solid line); curve b the split 1D BECs (green dashed line); and curve c, the merged 1D BEC (blue dotted line).

quence of the BdG equations present in the GPE equation. In spite of such fluctuations, the split and merged condensates present almost the same Thomas-Fermi density profiles for any temperature $T<T_{c}$.

From these observations, we conclude that in spite of the initial phase fluctuations in the quasicondensate regime at finite-temperature, there is a preservation of "phase coherence length" during the splitting and merging process, if adiabaticity is satisfied.

\section{SUMMARY}

Summarizing, we have discussed coherence effects in the splitting and merging of a $1 \mathrm{D}^{87} \mathrm{Rb} \mathrm{BEC}$. This is done by creating two spatially separated condensates from an initial 1D condensate by deforming the trapping potential into a double-well potential. We have analyzed the case of zero temperature as well as finite-temperature in the so-called quasi-condensate regime, where the phase coherence length is smaller than the size of the system. At zero temperature and for a process adiabatic enough where the splitting and merging times are much larger than the characteristic time of the system given by the inverse of the trap frequency $t_{\mathrm{sys}}=2 \pi / \omega_{z}$, a coherent splitting followed by a coherent merging is achieved and there is a constant relative phase between the initial and the final merged condensates. On the contrary if the splitting and merging times are not larger than the relevant time scale, the split condensates acquire a random relative phases and merging is no longer possible. In this case, the system acquires large density and phase 
fluctuations on a length scale comparable with the coherence length $l_{c}$, and a description based on the GPE becomes invalid. In the case of finite-temperature our results show that even in the presence of strong phase fluctuations, if the process of splitting and merging is carried out fully adiabatically, the condensate preserves the Thomas-Fermi density profile and there is phase coherence length preservation. In such cases, the merged condensate is a quasi-condensate with the same initial density profile as the initial condensate and with the same phase coherence length, although the "overall" phase of the quasi-condensate is not preserved. This situation occurs even at temperatures very near the critical value for condensation as long as the trapping potential remains in place. Our results may have a useful implication for manipulating 1D BECs at zero as well as finite-temperatures such as in atom lasers, interferometry, and solitons. In particular, our results agree qualitatively well with the recent measurements in Ref. 17].

\section{Acknowledgements}

A. M. wishes to thank the Deutscher Akademisher Austausch Dienst (DAAD) for financial support. We thank V. Ahufinger, J. Arlt, H. Kreutzmann, J. Schmiedmeyer, and M. K. Oberthaler for discussions. We acknowledge support from Deutsche Forschungsgemeinschaft (SFB 407, SPP1116, 432 POL, and GK 282), from the ESF Programme QUDEDIS, from the EU IP Programme SCALA, and the Spanish Grant No. MCYT BFM-2002-02588.

[1] M. H. Anderson, J. R. Ensher, M. R. Matthews, C. E. Wieman, and E. A. Cornell, Science 269, 198 (1995).

[2] K. B. Davis, M.-O. Mewes, M. R. Andrews, N. J. van Druten, D. S. Durfee, D. M. Kurn, and W. Ketterle, Phys. Rev. Lett. 75, 3969 (1995).

[3] C. C. Bradley, C. A. Sackett, J. J. Tollett, and R. G. Hulet, Phys. Rev. Lett. 75, 1687 (1995).

[4] D. G. Fried, T. C. Killian, L. Willmann, D. Landhuis, S. C. Moss, D. Kleppner, and T. J. Greytak, Phys. Rev. Lett. 81, 3811 (1998).

[5] A. Robert, O. Sirjean, A. Browaeys, J. Poupard, S. Nowak, D. Boiron, C. I. Westbrook, and A. Aspect, Science 292, 461 (2001).

[6] S. Jochim, M. Bartenstein, A. Altmeyer, G. Hendl, S. Riedl, C. Chin, J. H. Denschlag, and R. Grimm, Science 302, 2101 (2003).

[7] M. W. Zwierlein, C. A. Stan, C. H. Schunck, S. M. F. Raupach, S. Gupta, Z. Hadzibabic, and W. Ketterle, Phys. Rev. Lett. 91, 250401 (2003).

[8] M. Greiner, C. A. Regal, and D. S. Jin, Nature (London) 426, 537 (2003).
[9] M. Köhl, T. W. Hansch, and T. Esslinger, Phys. Rev. A. 65, 021606(R) (2002).

[10] P. W. H. Pinkse, T. Fischer, P. Maunz, and G. Rempe, Nature (London) 404, 365 (2000).

[11] A. P. Chikkatur, Y. Shin, A. E. Leanhardt, D. Kielpinski, E. Tsikata, T. L. Gustavson, D. E. Pritchard, and W. Ketterle, Science 296, 2193 (2002).

[12] T. H. Maiman, Nature (London) 187, 493, (1960).

[13] W. Yi and L.-M. Duan, Phys. Rev. A 72, 043607 (2005).

[14] Z. Hadzibabic, S. Stock, B. Battelier, V. Bretin, and J. Dalibard, Phys. Rev. Lett. 93, 180403 (2004).

[15] Y. Shin, M. Saba, T. A. Pasquini, W. Ketterle, D. E. Pritchard, and A. E. Leanhardt, Phys. Rev. Lett. 92, 050405 (2004).

[16] L. Pezzé, L. A. Collins, A. Smerzi, G. P. Berman, and A. R. Bishop, Phys. Rev. A 72, 043612 (2005).

[17] T. Schumm, S. Hofferberth, L. M. Andersson, S. Wildermuth, S. Groth, I. Bar-Joseph, J. Schmiedmayer, and P. Kruger, Nature 1, 57 (2005).

[18] J. Javanainen and M. Wilkens, Phys. Rev. Lett. 78, 4675 (1997).

[19] A. J. Leggett and F. Sols, Phys. Rev. Lett. 81, 1344 (1998).

[20] N. J. van Druten and W. Ketterle, Phys. Rev A 54, 656 (1996).

[21] D. S. Petrov, G. V. Shlyapnikov, and J. T. M. Walraven, Phys. Rev. Lett. 85, 3745 (2000).

[22] H. Moritz, T. Stöferle, M. Köhl, and T. Esslinger, Phys. Rev. Lett. 91, 250402 (2003).

[23] T. Stöferle, H. Moritz, C. Schori, M. Köhl, and T. Esslinger, Phys. Rev.Lett. 92, 130403 (2004).

[24] B. Paredes, A. Widera, V. Murg, O. Mandel, S. Fölling, I. Cirac, G. V. Shlyapnikov, and T. W. Hänsch, Nature (London) 429, 277 (2004).

[25] T. Kinoshita, T. Wenger, and D. S. Weiss, Science 305, 1125 (2004).

[26] A. Görlitz, J. M. Vogels, A. E. Leanhardt, C. Raman, T. L. Gustavson, J. R. Abo-Shaeer, A. P. Chikkatur, S. Gupta, S. Inouye, T. Rosenband, and W. Ketterle, Phys. Rev. Lett. 87, 130402 (2001).

[27] D. S. Petrov, M. Holzmann, and G. V. Shlyapnikov, Phys. Rev. Lett. 84, 2551 (2000).

[28] Yu. Kagan, V. A. Kashurnikov, A. V. Krasavin, N. V. Prokof'ev, and B. V. Svistunov, Phys. Rev. A 61, 43608 (2000).

[29] A. I. Safonov, S. A. Vasilyev, I. S. Yasnikov, I. I. Lukashevich, and S. Jaakkola, Phys. Rev. Lett. 81, 4545 (1998).

[30] Lev Pitaevskii and S. Strigari, Bose-Einstein Condensation (Oxford Science Publications, London, 2003).

[31] M. Girardeau, J. Math. Phys. 1, 516 (1960).

[32] C. Menotti and S. Stringari, Phys. Rev. A 66, 043610 (2002).

[33] K. V. Kheruntsyan, D. M. Gangardt, P. D. Drummond, and G. V. Shlyapnikov, Phys. Rev. Lett. 91, 040403 (2003).

[34] P. Pedri and L. Santos, Phys. Rev. Lett. 91, 110401 (2003).

[35] D. S. Petrov, G. V. Shlyapnikov, and J. T. M. Walraven, Phys. Rev. Lett. 87, 050404 (2001).

[36] S. Dettmer, D. Hellweg, P. Ryytty, J. J. Arlt, W. Ertmer, K. Sengstock, D. S. Petrov, G. V. Shlyapnikov, H. Kreutzmann, L. Santos, and M. Lewenstein, Phys. Rev. Lett. 87, 160406 (2001).

[37] H. Kreutzmann, A. Sanpera, L. Santos, M. Lewenstein, 
D. Hellweg, L. Cacciapuoti, M. Kottke, T. Schulte, K. Sengstock, J. J. Arlt, and W. Ertmer, Appl. Phys. B: Lasers Opt. 76, 165 (2003).

[38] D. Hellweg, S. Dettmer, P. Ryytty, J. J. Arlt, W. Ertmer, K. Sengstock, D. S. Petrov, G. V. Shlyapnikov, H. Kreutzmann, L. Santos, and M. Lewenstein, Appl. Phys. B: Lasers Opt. 73, 781 (2001).

[39] S. Richard, F. Gerbier, J. H. Thywissen, M. Hugbart, P. Bouyer, and A. Aspect, Phys. Rev. Lett. 91, 010405 (2003).

[40] F. Gerbier, J. H. Thywissen, S. Richard, M. Hugbart, P. Bouyer, and A. Aspect, Phys. Rev. A 67, 051602(R) (2003).

[41] D. Kadio, M. Gajda, and K. Rzążewski, Phys. Rev. A 72, 013607 (2005).

[42] F. Dalfovo and S. Giorgini, Rev. Mod. Phys. 71, 463 (1999).

[43] M. J. Davis, S. A. Morgan, and K. Burnett, Phys. Rev. Lett. 87, 160402 (2001).

[44] M. J. Davis, S. A. Morgan, and K. Burnett, Phys. Rev. A 66, 053618 (2002).

[45] M. J. Davis, R. J. Ballagh, and K. Burnett, J. Phys. B 34, 4487 (2001).

[46] M. J. Davis and S. A. Morgan, Phys. Rev. A 68, 053615
(2003).

[47] M. Brewczyk, P. Borowski, M. Gajda, and K. Rzążewski, J. Phys. B 37, 2725 (2004).

[48] K. Góral, M. Gajda, and K. Rzążewski, Opt. Express 8, 92 (2001).

[49] K. Góral, M. Gajda, and K. Rzążewski, Phys. Rev. A 66, 051602(R) (2002).

[50] H. Schmidt, K. Góral, F. Floegel, M. Gajda, and K. Rzążewski, J. Opt. B: Quantum Semiclassical Opt. 5, S96 (2003).

[51] R. Bach and K. Rzążewski, Phys. Rev. A 70, 063622 (2004).

[52] R. Bach and K. Rzążewski, Phys. Rev. Lett. 92, 200401 (2004).

[53] E. Altman, E. Demler, and M. D. Lukin, Phys. Rev. A 70, 013603 (2004).

[54] Y. Shin, C. Sanner, G.B. Jo, T.A. Pasquini, M. Saba, W. Ketterle, D.E. Pritchard, M. Vengalattore, and M. Prentiss, Phys. Rev. A 72, 021604(R) (2005).

[55] M. Olshanii, Phys. Rev. Lett. 81, 938 (1998).

[56] S. I. Shevchenko, Sov. J. Low. Temp. Phys. 18, 223 (1992). 